\section{PC-029 QUALITY OF GEMCITABINE READY TO ADMINISTER PREPARATIONS}

${ }^{1} \mathrm{R}$ Trittler*, ${ }^{2} \mathrm{H}$ Reinhardt, ${ }^{3} \mathrm{MJ}$ Hug. 'University Medical Centre, Pharmacy, Freiburg, Germany; ${ }^{2}$ University Medical Centre, Haematology, Freiburg, Germany; ${ }^{3}$ University Medical Centre, Apotheke, Freiburg, Germany

10.1136/ejhpharm-2019-eahpconf.110

Background Gemcitabine ready to administer bags are frequently prepared in hospital pharmacies. Long-term stability for 84 days is published ${ }^{1}$ and dose-banding is already established at some hospitals. Little is known about the quality of different gemcitabine preparations.

Purpose Dose-banding has the advantage of quality control before use. However, with increasing storage time bags may lose quality due to degradation products with unknown effects.

Material and methods We performed a stability study with ready to administer bags prepared from gemcitabine hexal concentrate in the hospital pharmacy aseptic production unit and with ready to administer bags from gemcitabine SUN. Therefore, we determined the gemcitabine concentration and analysed the degradation products 2'-Desoxy-2', 2'-difluoruridin (dFdU), O6,5'-Cyclo-5,6-dihydro-2'-desoxy-2', 2'-difluoruridin and two diastereomeric alcohols during a storage time of up to 84 days using HPLC/DAD and LC-MS. In addition, we measured the $\mathrm{pH}$ of all preparations at the beginning and after 84 days. All methods were validated following the guidelines for the practical stability studies of anti-cancer drugs. ${ }^{2}$

Results We could confirm stability of 84 days in all gemcitabine preparations. In all bags the gemcitabine concentration was $>95 \%$. The content of all tested degradation products was significantly higher in the bags produced by the pharmacy in $0.9 \% \mathrm{NaCl}$. In these bags the content of the degradation products increased significantly during storage time, whereas in the ready to administer bag gemcitabine SUN only a small amount of $\mathrm{dFdU}$ was found. Here the signal of $\mathrm{dFdU}$ did not increase during a storage time over 13 months. The $\mathrm{pH}$ was about 7.0, whereas the $\mathrm{pH}$ in the bags diluted from gemcitabine concentrate was 2.7 .

Conclusion We conclude that gemcitabine preparations with a more physiological $\mathrm{pH}$ have better quality. The advantages are longer stability with no increase of hydrolysis products over storage and the physiological $\mathrm{pH}$ may be more comfortable for the patient. To improve quality, we should consider changing the $\mathrm{pH}$ in the gemcitabine preparations in hospital pharmacies.

\section{REFERENCES AND/OR ACKNOWLEDGEMENTS}

1. http://www.esop.li/ejop.php

2. https://ejhp.bmj.com/content/19/3/278

No conflict of interest.

\section{PC-030 PHYSICAL, CHEMICAL AND MICROBIOLOGICAL STABILITY OF SIROLIMUS $0.4 \%$ IN TOPICAL FORMULATIONS}

${ }^{1} \mathrm{C}$ Cortell ${ }^{*},{ }^{1} \mathrm{MA}$ Martinez, ${ }^{1} \mathrm{C}$ Molina, ${ }^{1} \mathrm{AC}$ Cercos, ${ }^{1} \mathrm{M}$ Climente, ${ }^{2} \mathrm{~V}$ Merino. ${ }^{1}$ Hospital Doctor Peset, Pharmacy, Valencia, Spain; ${ }^{2}$ Universidad de Valencia, Pharmaceutical Technology, Valencia, Spain

10.1136/ejhpharm-2019-eahpconf.111
Background Facial angiofibromas in tuberous sclerosis are an aesthetic problem within the clinical context of the disease. There are currently few stability studies that allow selecting the best topical sirolimus therapy.

Purpose To improve the formulation of sirolimus $0.4 \%$ for the treatment of facial angiofibromas in tuberous sclerosis and to determine the period of validity (physical, chemical and microbiological stability) of the proposed formulations.

Material and methods Three formulations of sirolimus $0.4 \%$ were prepared (each in duplicate, A and B). Facilities and equipment: biological safety cabinet with individual protection equipment for the manipulator. Conditions: aluminum tubes, temperature $2^{\circ} \mathrm{C}-8^{\circ} \mathrm{C}$.

- Gel: sirolimus $0.4 \%, \quad$ transcutol $10 \%$, hydroxypropylmethylcellulose $2 \%$, water for injection (API: sterile solution) c.s.p. $20 \mathrm{~g}$.

- Ointment: sirolimus $0.4 \%$,transcutol $10 \%$, lanolin $10 \%$, shea butter 20\%, vitamin E 1\%, vaseline c.s.p. 20 g.

- Emulsion: sirolimus 0.4\%, transcutol 10\%, absorption base W/O 20\%, API c.s.p. 20 g.

Physical stability: $\mathrm{pH}$ was determined with reactive strips at $\mathrm{t}=15$ and 30 days. Properties of uniformity, extensibility, absence of crystals and absence of phase separations were evaluated on the transparent surface according to three levels: level 1, at least favourable and level 3, the most favourable.

Chemical stability: percentage content of remaining sirolimus (\%CR) in formulations $\mathrm{A}$ and $\mathrm{B}$ was determined at times $(t)=0.1$ and 2 days, and every 2 days until $t=30$ days, and t90 (sampling time at which it reached 90\% of $\%$ CR), when $\%$ CR was $\leq 90 \%$. Analytical method: high-performance liquid chromatography with ultraviolet/visible detection.

Microbiological stability: formulations $\mathrm{A}$ and $\mathrm{B}$ were incubated at $37^{\circ} \mathrm{C}$ on Mueller-Hinton agar and blood agar at $\mathrm{t}=15$ and 30 days.

Results Physical stability: $\mathrm{pH}$ did not change on days 15 and 30: 6.0 for the gel and the emulsion and 7.0 for the ointment.

- Gel: uniformity, level 3; extensibility, level 3; absence of crystals, level 3; absence of phase separations, level 3.

- Ointment: uniformity, level 3; extensibility, level 1; absence of crystals, level 3; absence of phase separations, level 3.

- c) Emulsion: uniformity, level 3; extensibility, level 2; absence of crystals, level 3; absence of phase separations, level 3.

Chemical stability: t90 was reached during the sampling period for the gel and emulsion formulations: $t 90=14$ and 2 days, respectively.

$\% \mathrm{CR}$ at $\mathrm{t}=30$ days was 110.4 (SD \pm 0.4 ) for the gel, 101.2 (SD \pm 4.6$)$ for the ointment and 87.7 (SD \pm 0.4$)$ for the emulsion.

Microbiological stability: cultures were negative for three formulations (A and $\mathrm{B}$ ) at $\mathrm{t}=15$ and 30 days.

Conclusion Each formulation has its own galenic characteristics that must be considered. Three formulations of sirolimus $0.4 \%$ maintain the physical and microbiological stability at 30 days at $2^{\circ} \mathrm{C}-8^{\circ} \mathrm{C}$. Only the ointment formulation maintains chemical stability, assigning a period of validity of 30 days at $2^{\circ} \mathrm{C}-8^{\circ} \mathrm{C}$

\section{REFERENCES AND/OR ACKNOWLEDGEMENTS}

No conflict of interest. 